\title{
Spatial Relationships in Choosing to Conserve Soil in Smallholder Rubber Farming
}

\author{
J. C. Edirisinghe ${ }^{1, \#}$ and W. Wijesuriya ${ }^{2}$ \\ ${ }^{1}$ Department of Agribusiness Management
}

Faculty of Agriculture and Plantation Management

Wayamba University of Sri Lanka

Makandura, Gonavila (NWP), Sri Lanka

${ }^{2}$ Rubber Research Institute of Sri Lanka, Dartonfield, Agalawatta, Sri Lanka

${ }^{\#}$ Corresponding Author

Tele: (94) 31229 9246; Fax: (94) 31229 9248; E-mail: jagathed@yahoo.com

\begin{abstract}
Soil conservation is one pivotal activity in Good Agricultural Practices. Thus, diffusing such activities among farming community is prudent. In spite of the fact that soil conservation choices are believed to be highly correlated in space, this aspect is seldom addressed in the literature. We take up this issue and attempt to model soil conservation choices of a sample of rubber farmers in Sri Lanka. Our focus is on one critical role in diffusion: the possibilities of interaction among farmers. Keeping in line with the previous literature, we deduce that individual farmers' decisions are often influenced by the choices and opinions of others in their immediate environment. The difficulty in capturing this effect is circumvented by estimating a spatial correlation coefficient in a spatial auto-regression framework. The dichotomous nature of the dependent variable forces us to utilize a 'Spatial Autoregressive Probit Model'. We employ recent developments in Bayesian econometric techniques in analysis. Results show a significant spatial correlation revealing a clear indication of a 'neighbourhood effect'.
\end{abstract}

KEYWORDS: Neighbourhood effect, Bayesian Spatial Autoregressive Probit

\section{Introduction}

A farmer's objective in using conservation practices may be to protect soil from eroding and to maintain high nutritional status for a longer time. This leads to a reduction in the amount of fertilizer applied artificially to soil, reducing costs of production, finally leading to higher levels of profits. Such practices therefore, 
benefit farmers directly through their impacts on profits and benefit the society as a whole through its impacts on environmental sustainability. Therefore, many have studied the adoption of conservation practices with the objective on finding ways to improve diffusion of this importance technology among the farming community in many countries. In his meta-analysis of adoption of agro forestry practices in a decade of research studies, Pattanayak et al. (2003) describes five major categories that have been studied as influencing adoption in soil and water conservation: preferences, resource endowments, market incentives, biophysical factors and risk. This is in no exception with other adoption studies on agriculture in general in developing countries (Feder et al., 1985). These studies, have omitted one important aspect in diffusion: the ability of farmers to learn from each other. The neighborhood provides an important source of social capital. Especially in the case of 'voting behavior', there is strong circumstantial evidence on the existence of neighborhood effects (Propper et al., 2005).

Influence of one's choice on another is difficult to measure. Spatial econometrics literature provides a way around this problem. Notable recent studies that use spatial econometrics to measure the effect of neighbors on the choices made include among others, the study on adoption of rice varieties in Bangladesh by Holloway et al. (2002), investigation of selling decisions by dairy farmers by Holloway and Lapar (2007), and business recovery in the aftermath of hurricane Katrina by LeSage et al. (2010).

In this study, we attempt to model the adoption of conservation practices, taking soil conservation as an example, and measure the effects of the neighborhood, using recent developments in Bayesian econometrics.

\section{Methods}

\section{Model}

Adoption of a particular agronomic practice or a technology depends on the returns or the expected returns due to adoption. In agronomic practices such as soil conservation, the returns are difficult to measure. The difficulty that occurs is twofold. First, the returns are not immediate and occur on a longer time span. Second, it is difficult to measure the returns from only one agronomic practice in the light of several factors that contribute to returns in agriculture. However, the ordinal utility theory states that utility from two goods, although cannot be measured with an economic meaning, can be ordered such that a rational person may select the good with higher utility over the other. On the same note, assume that there is some utility $\left(\boldsymbol{U}_{j i}\right)$ derived by $i^{\text {th }}$ farmer (for $\boldsymbol{i}=\mathbf{1}, \ldots \ldots, \boldsymbol{N}$ ) due to adoption of technology, $j$ (for $j=0,1$ ). The farmer will adopt this technology (make the choice 1) if, $\boldsymbol{U}_{j i} \geq \boldsymbol{U}_{0 i}$ where, $\boldsymbol{U}_{0 i}$ is the level of utility with no adoption. 
Assume that $\boldsymbol{U}_{j i}$ and $\boldsymbol{U}_{\mathbf{0} i}$ are normally distributed. Then, the difference $\boldsymbol{U}_{j i}-\boldsymbol{U}_{\mathbf{0 i}}$ will also be normally distributed. Because we do not observe this utility difference, we can define it in terms of a latent distribution, $\boldsymbol{z}_{\boldsymbol{i}}=\boldsymbol{U}_{1 i}-\boldsymbol{U}_{\mathbf{0 i}}$. Although, we do not observe this latent distribution, we observe whether farmer adopts soil conservation practices or not. We relate this unobserved utility to a set of covariates expected to be associated with the choice of soil conservation as;

$$
z_{i}=x_{i} \beta+e_{i}
$$

The relationship between the latent variable $z$ and the observed $\boldsymbol{y}_{\boldsymbol{i}}$ take the form

$$
\begin{aligned}
& y_{i}=1 \text { if } z \geq 0 \\
& y_{i}=0 \text { if } z<0
\end{aligned}
$$

Following LeSage and Pace (2009), we incorporate a spatial autoregressive component into (1) in order to capture the neighborhood effect on adoption decision.

$$
z=\rho W z+X \beta+e \quad e^{\sim} f^{N}\left(0, I_{N}\right)
$$

Where, $\mathrm{Wz}$ is the spatial lag dependent variable and $\mathrm{W}$ is an $\mathbf{N} \times \mathbf{N}$ spatial weight matrix. The elements of this matrix are either $\mathbf{1} / \boldsymbol{m}$ or 0 where $\mathrm{m}$ is some number of nearest neighbors. All elements in the $\mathrm{i}^{\text {th }}$ row of the matrix that are not associated with neighboring observations take the values of 0 . Therefore, on the way that $\mathrm{W}$ is constructed, it is row stochastic (non-negative and each row sums to one). Because of this reason, the $\mathrm{N} \times \mathbf{1}$ vector, $\mathrm{Wz}$ consists of average of the utility of $m$ neighboring farmers. This creates the mechanism for modeling interdependence of famer choice of adopting soil conservation practice. The scalar parameter $\boldsymbol{\rho}$ measures the strength of this dependence (neighborhood effect). If $\boldsymbol{\rho}=\mathbf{0}$, there is no dependence.

We use Bayesian methods in estimating the model in (3). To implement these models in a Bayesian framework, a prior pdf over parameters, $\boldsymbol{\Pi}(\boldsymbol{\theta})$ and likelihood, $\boldsymbol{f}(\mathbf{y} \mid \boldsymbol{\theta})$ is formed and the posterior distribution $\boldsymbol{f}(\boldsymbol{\theta} \mid \mathbf{y})$ for the parameters is studied with the usual relationship in Bayesian analysis: $\boldsymbol{\pi}(\boldsymbol{\theta} \mid \mathbf{y}) \propto \boldsymbol{f}(\mathbf{y} \mid \boldsymbol{\theta}) \boldsymbol{\pi}(\boldsymbol{\theta})$. Parameter vector, $\boldsymbol{\theta}$ include the unknown coefficients $\boldsymbol{\beta}$ and the latent variable, $\mathrm{z}$ is estimated alongside regression coefficients as Gibbs sampling with data augmentation 
following Albert and Chib (1993). As the prior information on the shape of $\boldsymbol{\rho}$ and $\boldsymbol{\beta}$ are vague, we use diffuse priors on these. Thus, the posterior has the form;

$\pi\left(\theta \mid y_{,} z\right) \propto \prod_{i=1}^{W} f^{T N+}\left(z_{i} \mid \boldsymbol{\beta}_{i}\right)^{y_{1}} \times f^{T N-}\left(z_{i} \mid \boldsymbol{\beta}_{i}\right)^{\left(1-y_{i}\right)} \times \pi\left(\rho \mid \rho_{0}, \sigma_{0}\right) \times \pi\left(\boldsymbol{\beta} \mid \boldsymbol{\beta}_{0}, \boldsymbol{C}_{0}\right)$

We obtain the conditional distributions for $\beta$ and $\mathrm{z}$ from (4) which has the form:

$$
\begin{gathered}
\beta \mid \rho, y, z \sim f^{M N}\left(\widehat{\beta}, \operatorname{Cov}_{\hat{\beta}}\right) \\
z \mid \rho, y, \beta \sim f^{T N}\left(\widehat{z}, \operatorname{Cov}_{\hat{z}}\right)
\end{gathered}
$$

Where, $\ddot{\beta}=\left(X^{s} X+C_{0}^{-1}\right)^{-1}\left(X^{s}\left(I_{N}-\rho W\right) Z+C_{0}^{-1} \beta_{0}\right) ; \quad \operatorname{Cov}_{\hat{\beta}}^{\hat{\beta}}=\left(X^{s} X+C_{0}^{-1}\right)^{-1}$;

$\hat{\mathrm{z}}=\left(\left(\mathrm{I}_{\mathrm{N}}-\rho W\right)^{s}\left(\mathrm{I}_{\mathrm{N}}-\rho W\right)\right)^{-1}\left(\mathrm{I}_{\mathrm{N}}-\rho W\right)^{s} \mathrm{X} \beta ; \operatorname{Cov}_{\hat{\mathrm{z}}}=\left(\left(\mathrm{I}_{\mathrm{N}}-\rho W\right)^{s}\left(\mathrm{I}_{\mathrm{N}}-\rho W\right)\right)^{-1}$.

We draw from these conditional distributions following the Gibbs sampling procedure. It is difficult to find a suitable conditional for $\rho$ and therefore, the draw for $\rho$ follow a Random Walk Metropolis Hastings Algorithm.

Estimation is carried out using Matlab R2009a software. We use a burn in phase of 1000 and a results phase of 10000 . The convergence is assessed through studying trace plots.

\section{Data}

Data are from the Rubber Research Institute of Sri Lanka collected from the Moneragala district in Sri Lanka. A total of 262 smallholder rubber farmers are in the sample. Data collection was through face to face interviews using a pre-tested questionnaire that included questions on socio economic conditions of households and many agronomic practices in rubber cultivation, including soil conservation. We use presence of stone terraces as the main soil conservation techniques which is used to control soil erosion in rubber lands. For covariates, we use four variables related to human capital in the household (education, age and gender of household head and family size), four variables related to land and soil (topography, 3 dummies representing 4 soil types and land extent), and two variables relating to other activities in the land (presence of intercrops and cover crops).

Data were not collected with the objective of a spatial analysis. Therefore, spatial co ordinance of individual households were not available. We used spatial co ordinance of centroids of Grama Niladari (GN) divisions to identify the locations of farmers in deciding the neighbors in the spatial weight matrix. 


\section{Results}

\section{Summary Statistics}

Summary statistics of adopters and non-adopters are recorded in Table 1. Percentage of females in both adopters and non-adopters are high. Distribution of mature lands and education in both groups are similar. However, distribution of sloping lands in the two groups is different. Higher percentage of adopters has lands with higher slopes. The percentages of farmers who have grown intercrops are similarly distributed between adopters and non-adopters. In the case of cover crops, the distribution among the two groups is similar as well.

Table 1: Summary statistics by adoption status

\begin{tabular}{cccc}
\hline Variable & & Non-Adopters & Adopters \\
\hline \multirow{2}{*}{ Gender } & Male & 16.67 & 15.60 \\
& Female & 83.33 & 84.40 \\
\hline \multirow{2}{*}{ Maturity } & Mature & 62.70 & 63.83 \\
& Immature & 37.30 & 36.17 \\
\hline \multirow{2}{*}{ Education } & Primary & 55.79 & 61.65 \\
& Secondary & 33.06 & 30.83 \\
& O/L & 8.68 & 6.02 \\
& A/L & 2.48 & 1.50 \\
\hline \multirow{2}{*}{ Topography } & Flat & 39.68 & 8.51 \\
& Medium & 58.33 & 80.85 \\
& High Slope & 1.98 & 10.64 \\
\hline Intercrops & Yes & 37.30 & 28.37 \\
& No & 62.70 & 71.63 \\
\hline Cover crops & Yes & 87.70 & 91.49 \\
& No & 12.30 & 8.51 \\
\hline Mean family size & - & $4.45(1.434)$ & $4.48(1.339)$ \\
Mean age & - & $46.86(10.865)$ & $48.45(11.951)$ \\
Mean extent & - & $1.69(2.164)$ & $1.55(1.088)$ \\
\hline
\end{tabular}

Standard deviations are given within parentheses

\section{Spatial Autoregressive Probit Output}

Trace plots show convergence after 10000 iterations (Figure 01) and the effect of starting values of the Markov Chain has worn off. The posterior estimates of SARP model is reported in Table 2. Topography, the presence of intercrops and cover crops show a significant impact on adoption of soil conservation measures. Data fails to show a significant impact of any of the socio economic variables on adoption. 


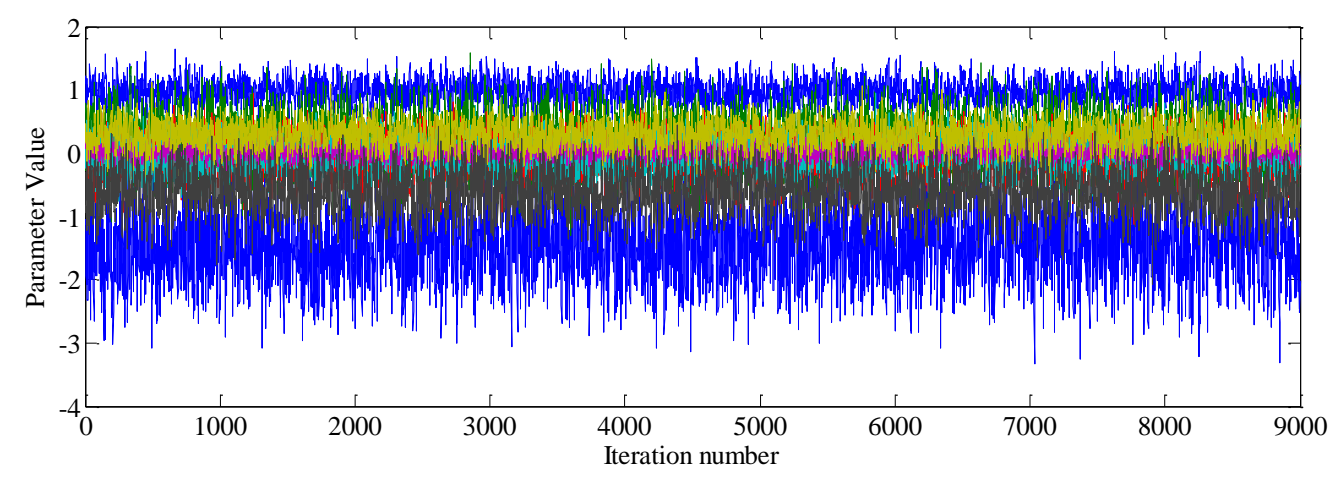

Figure 1: Trace plots of Gibbs sample draws for regression parameters

Table 2: Coefficient estimates of the Bayesian spatial autoregressive model

\begin{tabular}{cccc}
\hline Variable & Coefficient & $\begin{array}{c}\text { Standard } \\
\text { Deviation }\end{array}$ & p-level \\
\hline Constant & $-1.448^{*}$ & 0.528 & 0.001 \\
Maturity & 0.185 & 0.194 & 0.172 \\
Family Size & 0.040 & 0.061 & 0.256 \\
Gender & -0.010 & 0.227 & 0.482 \\
Age & 0.002 & 0.008 & 0.406 \\
Education & -0.075 & 0.121 & 0.266 \\
Extent & 0.041 & 0.076 & 0.290 \\
Topography & $0.976^{*}$ & 0.178 & 0.000 \\
Sandy & 0.204 & 0.391 & 0.294 \\
Clay & -0.049 & 0.262 & 0.426 \\
Gravel & -0.030 & 0.227 & 0.447 \\
Ownership & 0.013 & 0.097 & 0.439 \\
Intercrop & $0.287^{* *}$ & 0.193 & 0.068 \\
Cover crop & $-0.607^{*}$ & 0.312 & 0.024 \\
Rho & $0.621^{*}$ & 0.074 & 0.000 \\
\hline
\end{tabular}

However, important to note is the negative sign reported in education variable. Although, the expectation of the effect of education is positive, previous research on technology adoption has showed that education can be negative. The postulate is that on one hand education can increase adoption by lowering learning costs. On the other hand, it can discourage adoption as because it enable farmers to attract more off farm employment (Holloway et al., 2002).

Topography was given a score of 0 to 2 on the increasing slope of the land. A score of 0 meant the land is flat. Thus, the positive result is as expected. Higher the slope, higher is the probability of adoption of soil conservation practices. Intercropping has a positive significant impact on adoption while cover crops have a negative 
impact. Cover crops, although grown as a moisture conservation measure, has an impact on soil conservation as well. Therefore, the negative sign can be expected.

The spatial correlation coefficient $\rho$ returns a value of 0.62 and is significant at 5 percent error level. Very little density resides on the negative part of the real line in Figure 02 . As noted before, by construction of the matrix $\mathrm{W}$, the spatially lagged variable $\mathrm{Wz}$ represents the average of the utility of $m$ neighbouring farmers. Therefore, the estimate 0.62 represents a relatively strong relationship of one farmer's choice to adopt soil conservation practices on the average utility derived by neighbouring farmers. The positive sign of this correlation coefficient indicate a positive influence of the neighbours on the decisions of farmers.

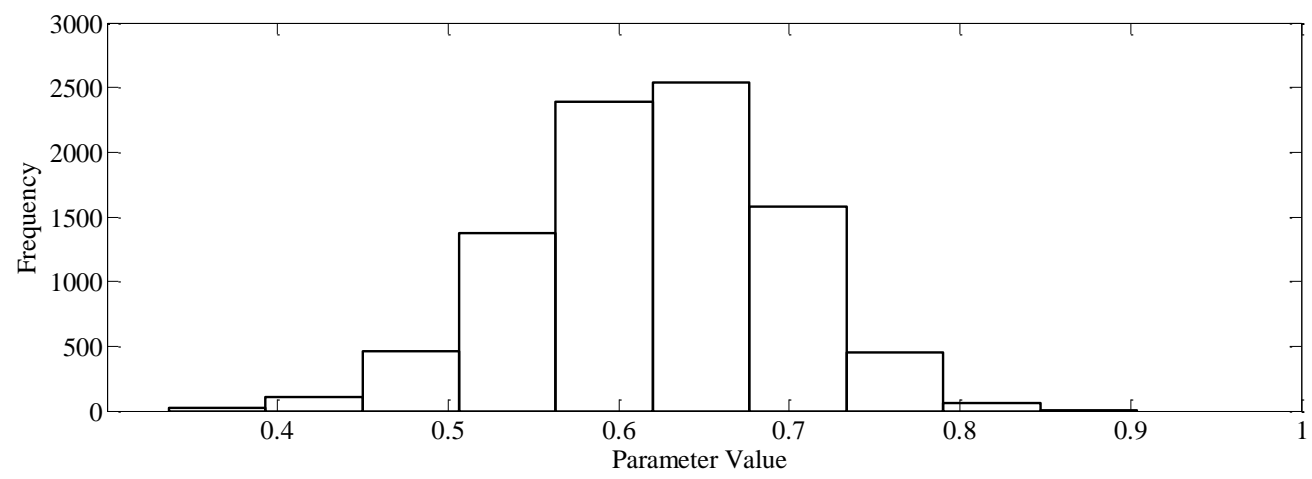

Figure 02: Random walk metropolis draws for spatial correlation coefficients

\section{Conclusions}

This study evaluated the impact of neighbourhood effect on farmer decisions on adoption of technology, taking soil conservation as an example. The employed spatial auto regressive model shows a strong, significant positive correlation on spatial auto regressive coefficient. This confirms our hypothesis of neighbourhood effects in dissemination of the soil conservation technology studied. This has implications on designing extension services to popularize such techniques in agriculture.

\section{Acknowledgement}

The financial assistance from the National Science Foundation of Sri Lanka is gracefully acknowledged. We are also indebted to all who supported in collecting data and the farmers who willingly provided information to make this study a success.

\section{References}

Albert, J. H. and S. Chib (1993). "Bayesian analysis of binary and polychotomous response data" Journal of the American Statistical Association, 88: 669-679. 
Feder, G., R. E. Just and D. Zilberman (1985). "Adoption of agricultural innovations in developing countries: A Survey" Economic Development \& Cultural Change, 33: 255 .

Holloway, G. and M. L. A. Lapar (2007). "How big is your neighbourhood? Spatial implications of market participation among Filipino smallholders" Journal of Agricultural Economics, 58: 37-60.

Holloway, G., B. Shankar and S. Rahmanb (2002). "Bayesian spatial probit estimation: a primer and an application to HYV rice adoption" Agricultural Economics, 27: 383402.

LeSage, J. P. and R. K. Pace (2009). "Introduction to spatial econometrics: Chapman \& Hall/CRC".

LeSage, J. P., R. K. Pace, N. Lam, R. Campanella and X. Liu (2010). "New Orleans business recovery in the aftermath of hurricane Katrina" Texas State UniversitySan Marcos.

Pattanayak, S. K., D. E. Mercer, E. O. Sills, J. C. Yang and K. Cassingham (2003). "Taking stock of agroforestry adoption studies" Agroforestry Systems, 57: 173-186.

Propper, C., R. Sarker, K. Jones, A. Bolster and S. Burgess (2005). "Neighbourhood social capital and neighbourhood effects" Environment and Planning, A 37: 1443. 